\title{
Numerical MOdel OF A THERMOACOUSTIC ENGINE
}

\author{
Omar Hireche $^{1}$, Catherine Weisman ${ }^{1,2}$, Diana Baltean-Carlès ${ }^{1,2}$, Maurice X. François ${ }^{1,2}$, Patrick Le Quéré ${ }^{1}$ and Luc \\ Bauwens $^{3}$ \\ ${ }^{1}$ Laboratoire d'Informatique pour la Mécanique et les Sciences de l'Ingénieur (LIMSI-CNRS), BP 133, F-91403, Orsay, \\ France \\ ${ }^{2}$ Université Pierre et Marie Curie, 4 Place Jussieu, F-75252, Paris Cedex 05, France \\ ${ }^{3}$ Department of Mechanical and Manufacturing Engineering, University of Calgary, Calgary, Canada T2N 1N4
}

\section{INTRODUCTION}

An asymptotically consistent small Mach number model of a complete thermoacoustic engine has been developed. The model couples linear acoustics in the resonator with a low Mach number flow model for the acoustically compact assembly stack t heat exchangers.

\section{PHYSICAL MODEL}

Both linear acoustics and incompressible flow are low Mach number approximations to the equations of gas dynamics. However the former assumes length and time in a ratio of the order of the speed of sound while the latter assumes a ratio comparable with the fluid velocity. For a common time scale, the two models assume length scales in the order of the Mach number $M$. Additionally [1-3], for the latter, a low Mach number approximation can be derived allowing for arbitrary spatially uniform pressure fluctuations that are superimposed to the dynamic correction of order $M^{2}$ that characterizes incompressible flow. An asymptotically consistent model of a complete thermoacoustic engine can be constructed matching these two approximations. Geometry is described in Fig. 1.

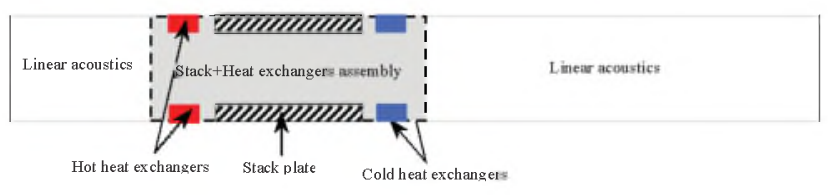

Fig. 1. System geometry

\section{ACOUSTICS}

The Riemann variables $L(x, t)=p^{\prime}-\rho u c$ and $R(x, t)=p^{\prime}+\rho u c$, in which $u$ is the longitudinal velocity, $T$ and $\rho$, leading order temperature and density, and $p^{\prime}$ the order $M$ acoustic pressure correction are introduced. $L$ and $R$ are constant respectively on characteristics moving left and right at the speed of sound c. In the resonator, $u$ and $p^{\prime}$ vary in space and time, while $p$ is constant. $\rho$ and $T$ are constant but with different values on the two sides of the heat exchangers. Taking the outer boundary conditions into account, $L$ at the left end of the heat exchanger section at time $t$ is related to $R$ at the same location at $\mathrm{t}$ minus the round-trip time. Likewise $R$ at the right end of the heat exchangers depends upon the previous value of $L$ at that end. This results in two relationships between acoustic pressure in the heat exchangers to the velocities at the ends.

\section{STACK AND HEAT EXCHANGERS}

In the stack and heat exchangers, calling $\boldsymbol{u}$ the velocity vector, and $p "(\mathbf{x}, t)$ the order $M^{2}$ dynamic pressure correction, the multidimensional flow is represented by the conservation equations for mass, momentum and energy:

$$
\begin{aligned}
& \frac{\partial \rho}{\partial t}+\nabla \cdot(\rho u)=0 \\
& \rho \frac{\partial u}{\partial t}+\rho u \cdot \nabla u=-\nabla p^{\prime \prime}+\nabla \cdot \tau \\
& \frac{\partial p}{\partial t}+\gamma \nabla \cdot(u p)=\nabla \cdot(k \nabla T)
\end{aligned}
$$

where $k$ is the thermal conductivity, $\gamma$ is the ratio of specific heats and $\tau$ is the stress tensor, related to velocity in the usual way for Newtonian fluids. Pressure, density and temperature are related by the equation of state, $p=\rho(\mathbf{x}, t) R T(\mathbf{x}, t)$. While this formulation supports timedependent pressure at leading order, the linear acoustics model in the resonator will only support fluctuations $p^{\prime}$ at order $M$, to which the heat exchanger section is transparent. Leading order pressure $p$ is an absolute constant, which results in further simplification in the energy equation. The heat exchanger model is completed by conservation of energy in the stack, which is governed by a standard heat equation. At the boundary, temperature and the heat flux vector are continuous.

The solution is obtained using a code derived from [4], that is second order accurate in time and space. Viscous and conductive terms are dealt with using an implicit formulation, while advective terms are explicit. Time integration uses a predictor-corrector formulation.

\section{MATCHING}

Acoustics provide two equations relating acoustic pressure in the compact heat exchanger section to the velocities at the two interfaces. Integration of the energy equation in the heat exchangers (above) over the heat exchangers computational domain results in a third one, relating the velocities at the two ends to heat transfer. Thus, while it is transparent to the acoustic pressure, the heat exchanger section appears in the acoustics as a point source of volume. Solving the three equations completes the acoustic formulation and, at each time step, provides boundary conditions to the numerical low Mach number flow model

\section{RESULTS}

Results were obtained for a resonator length of $8 \mathrm{~m}$ and a stack $0.15 \mathrm{~m}$ long, located at $1 \mathrm{~m}$ from the warm end. Cold end temperature was $293 \mathrm{~K}$. The fluid was helium at $1 \mathrm{MPa}$. 
The stack was made of stainless steel 304L, with thickness $0.2 \mathrm{~mm}$ and distance between plates, $0.77 \mathrm{~mm}$. Temperature ratios $\mathrm{T}_{\text {hot }} / \mathrm{T}_{\text {Cold }}$ of 1.3 and 2 were considered. Figure 2 shows the pressure history, while Fig. 3 shows velocities and Fig. 4, the phase relationships, for temperature ratio 1.3 and a standing wave as initial condition. Figures 5 and 6 refer to a temperature ratio of 2 , respectively for a standing wave and for resonant noise as initial conditions. A resolution of 34 by 1024 grid points was used in space and of 628 time steps/period.

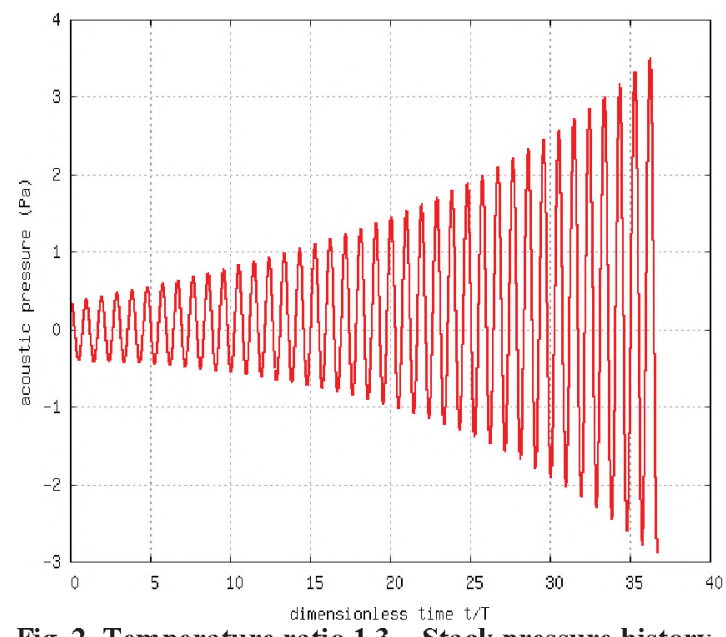

Fig. 2. Temperature ratio 1.3 - Stack pressure history

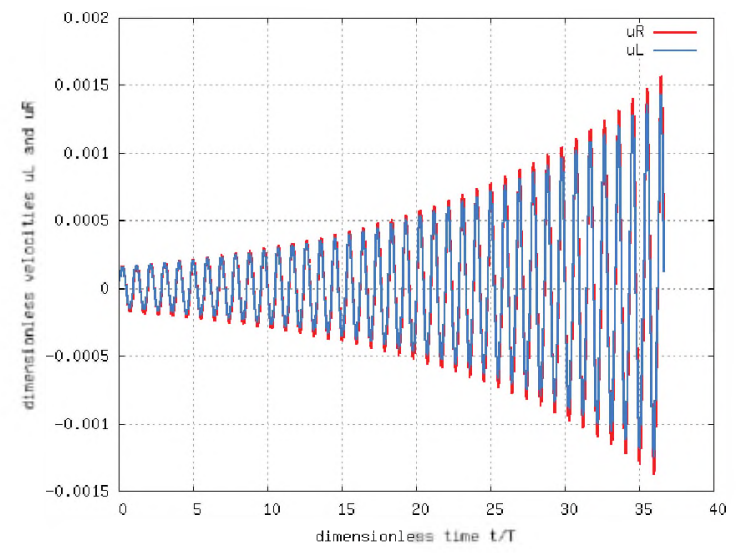

Fig. 3. Temperature ratio 1.3 - Stack end velocities

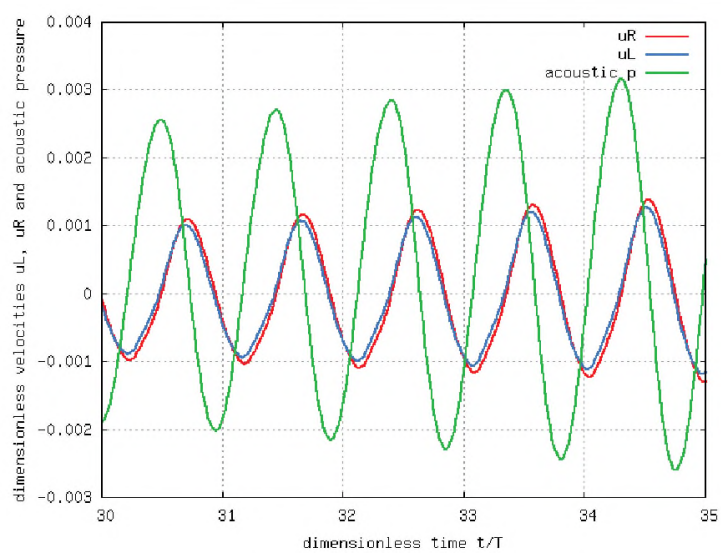

Fig. 4. Temperature ratio 1.3 - Phase relationships.
The results on Figs. 2 to 4 show that for lower temperature ratio, the lowest resonant mode is the most unstable; growth rate is $3.2 \mathrm{~s}^{-1}$. However, for the higher temperature ratio, the second mode manifests itself and grows faster, regardless of whether the system was excited by random noise or by the resonant mode. Starting with noise, growth rates are respectively $8 \mathrm{~s}^{-1}$ and $13.5 \mathrm{~s}^{-1}$.

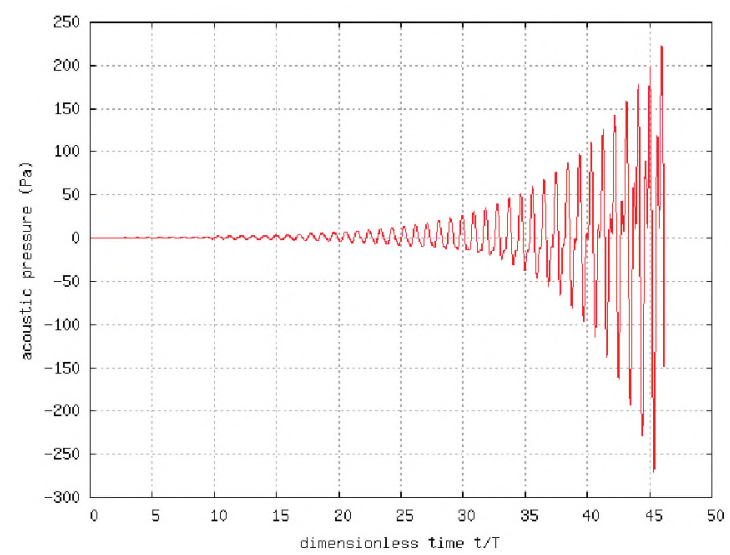

Fig. 5. Temperature ratio 2 - Initiation with standing wave.

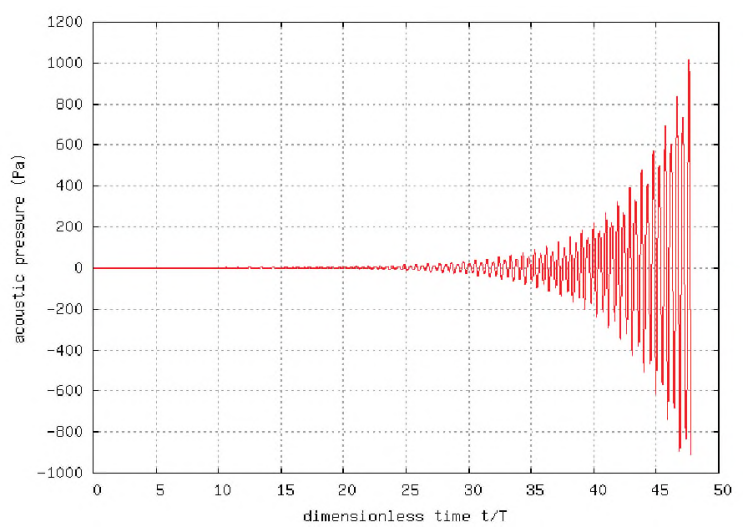

Fig. 6. Temperature ratio 2 - Initiation with random noise.

\section{REFERENCES}

[1]S. Paolucei. (1982) On the filtering of sound from the NavierStokes equations. Sandia National Laboratories report SAND828257.

[2]A. Majda and J.A. Sethian. (1984) The derivation and numerical solution of the equations for zero Mach number combustion. Combust. Sci. Tech. 42, 185-205.

[3]L. Bauwens. (1996). Oscillating flow of a heat-conducting fluid in a narrow tube. J. Fluid Mech., 324, 135-161.

[4]P. Le Quéré, C. Weisman, H. Paillère, J. Vierendeels, E. Dick, R. Becker, M. Braack and J. Locke. (2005) Modelling of Natural Convection Flows with large Temperature Differences: A Benchmark Problem for Low Mach Number Flow, Part 1. Reference Solutions. ESAIM: M2AN, 39 (3), 609-616.

\section{ACKNOWLEDGMENTS}

Support from the Natural Science and Engineering Research Council of Canada is gratefully acknowledged.

The computations were performed on the NEC SX8 of the CNRS-IDRIS Computing Center 